\title{
Discovery of directional and nondirectional pioneer transcription factors by modeling DNase profile magnitude and shape
}

\author{
Richard I Sherwood ${ }^{1,4}$, Tatsunori Hashimoto ${ }^{2,4}$, Charles W O’Donnell ${ }^{2-4}$, Sophia Lewis ${ }^{1}$, Amira A Barkal ${ }^{1}$, \\ John Peter van Hoff ${ }^{1}$, Vivek Karun ${ }^{1}$, Tommi Jaakkola² \& David K Gifford ${ }^{2,3}$
}

\begin{abstract}
We describe protein interaction quantitation (PIQ), a computational method for modeling the magnitude and shape of genome-wide DNase I hypersensitivity profiles to identify transcription factor (TF) binding sites. Through the use of machine-learning techniques, PIQ identified binding sites for $>700 \mathrm{TFs}$ from one DNase I hypersensitivity analysis followed by sequencing (DNase-seq) experiment with accuracy comparable to that of chromatin immunoprecipitation followed by sequencing (ChIP-seq). We applied PIQ to analyze

DNase-seq data from mouse embryonic stem cells differentiating into prepancreatic and intestinal endoderm. We identified 120 and experimentally validated eight 'pioneer' TF families that dynamically open chromatin. Four pioneer TF families only opened chromatin in one direction from their motifs. Furthermore, we identified 'settler' TFs whose genomic binding is principally governed by proximity to open chromatin. Our results support a model of hierarchical TF binding in which directional and nondirectional pioneer activity shapes the chromatin landscape for population by settler TFs.
\end{abstract}

Manipulation of TFs can reprogram cellular identity ${ }^{1,2}$ and rewire intercellular signaling pathways ${ }^{3,4}$. Efforts to predict TF binding patterns have been hampered by incomplete understanding of the rules governing the choice of TF binding sites. Highly accurate genomewide methods have been developed to localize the condition-specific binding of TFs to the genome, facilitating the elucidation of genome regulatory elements and gene regulatory networks ${ }^{5,6}$. Chromatin immunoprecipitation of selected protein-DNA complexes followed by high-throughput sequencing and mapping of the immunoprecipitated DNA (ChIP-seq) $)^{7}$ has become a valued method for analysis of TF locations and can reliably identify where TFs bind genome-wide

\footnotetext{
${ }^{1}$ Division of Genetics, Department of Medicine, Brigham and Women's Hospital and Harvard Medical School, Boston, Massachusetts, USA. ${ }^{2}$ Computer Science and Artificial Intelligence Laboratory, Massachusetts Institute of Technology, Cambridge, Massachusetts, USA. ${ }^{3}$ Department of Stem Cell and Regenerative Biology, Harvard University and Harvard Medical School, Cambridge, Massachusetts, USA. ${ }^{4}$ These authors contributed equally to this work. Correspondence should be addressed to D.K.G. (gifford@mit.edu) or R.I.S. (rsherwood@partners.org).

Received 13 March 2013; accepted 16 December 2013; published online 19 January 2014; doi:10.1038/nbt.2798
}

within 10 base pairs (bp) $)^{8,9}$. In each ChIP-seq experiment, a single $\mathrm{TF}$ is profiled, and this requires either an antibody specific to the $\mathrm{TF}$ or the incorporation of a tag into the TF being profiled. DNaseseq $^{10}$ is an assay that takes advantage of the preferential cutting of DNase I in open chromatin ${ }^{11}$ and the steric blockage of DNase I by tightly bound TFs that protect associated genomic DNA sequences ${ }^{12}$. After deep sequencing of DNase I-digested genomic DNA from intact nuclei, genome-wide data on chromatin accessibility as well as TF-specific DNase I protection profiles that reveal the genomic binding locations of a majority of TFs are obtained ${ }^{13-16}$. Such TF signature 'DNase profiles' reflect the effect of the TF on DNA shape and local chromatin architecture, extending hundreds of base pairs from a TF binding site, and these profiles are centered on 'DNase footprints' at the binding motif itself, which reflects the biophysics of proteinDNA binding $15,17,18$. As DNase-seq experiments are TF-independent and do not require antibodies, it is possible to predict the binding of hundreds of different TFs to their genomic motifs from a single DNase-seq experiment. Several groups have developed algorithms to infer TF binding from DNase-seq data ${ }^{13,15,17-19}$, but these existing methods do not model TF-dependent chromatin accessibility well.

Here we aimed to improve on these methods conceptually in two ways. First, we take into account how individual TFs contribute to both the magnitude and spatial pattern of DNase I hypersensitivity. Not only does this improve our ability to identify binding of all TFs regardless of their DNase profiles, it also allows us to probe whether a factor increases local hypersensitivity. Second, we carefully integrate prior information, such as the quality of a motif match, so that the method behaves robustly even with weak motifs or low-coverage data.

\section{RESULTS}

\section{Protein interaction quantitation}

PIQ is a method for analyzing genome-wide DNase I hypersensitivity data. The input for PIQ is data from one or more DNase-seq experiments, the genome sequence of the organism assayed and a list of motifs represented as position weight matrices (PWMs) that describe candidate TF binding sites. PIQ uses machine-learning methods to normalize input DNase-seq data and then predicts TF binding by detecting both the shape and magnitude of DNase profiles ${ }^{15}$ specific to each TF (Fig. 1). The output of PIQ is the probability of occupancy for each candidate binding site in the genome, along with aggregate 
Input DNase-seq (one or multiple related experiments)

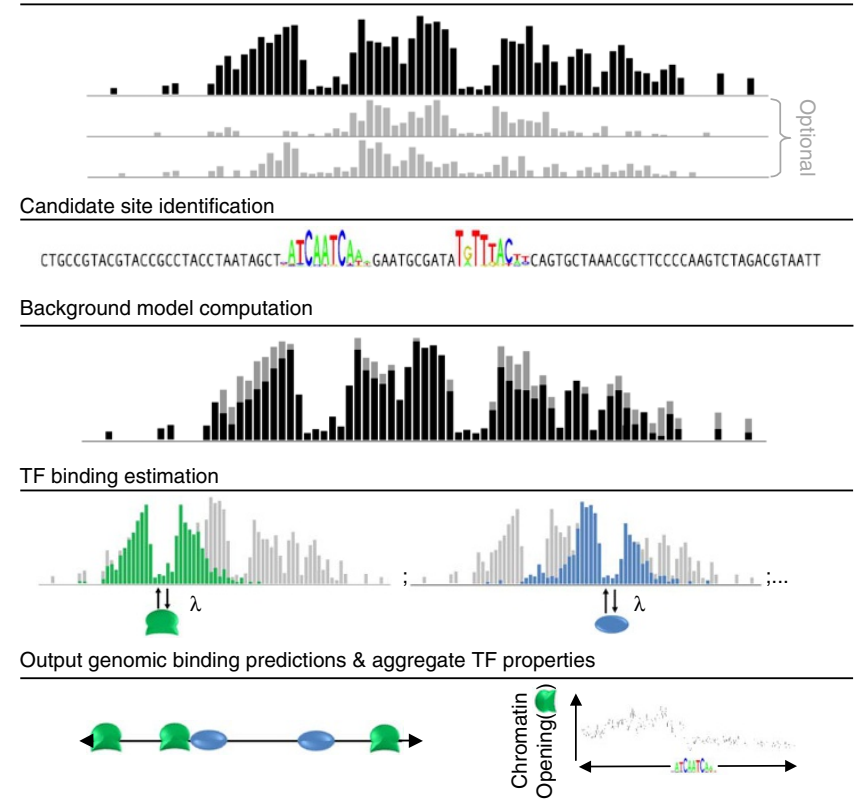

Figure 1 Accurate detection of dynamic TF binding using DNase-seq and $P I Q$. Schematic outlining the PIQ algorithm.

TF-specific scores (for example, metrics for TF-specific chromatin opening). For the results described in this paper, PIQ outputs protein binding at the locations of 733 TF motifs (after postprocessing; see below).

The PIQ algorithm consists of three steps: identification of a candidate site, computation of a background model and estimation of TF binding (Fig. 1).

In the first step, PIQ scans for DNase profiles at PWM motifs for 1,331 TFs derived from the JASPAR, UniPROBE and TRANSFAC databases $^{9-11}$ (see Supplementary Methods for explanation of motif choice). We choose to scan potentially bound motifs from the information in these databases and subsequently determine whether each site has a profile ${ }^{8}$, instead of detecting genome-wide footprints de novo and subsequently matching them to underlying motifs ${ }^{4-7}$, because motif-centered searching can take into account each TF's unique signature DNase profile information that is learned in subsequent steps of PIQ (Supplementary Fig. 1). This motif-specific information about the expected DNase I hypersensitivity profile surrounding a bound site improves individual binding prediction and allows complex enhancer and promoter profile clusters to more easily be deconvolved into a set of bound motifs, each imparting its signature profile on the chromatin.

In the second step, PIQ performs smoothing of the raw reads from each DNase-seq experiment to produce a robust foundation for profile detection. PIQ models raw DNase-seq reads as arising from a Gaussian process, which is a statistical model that removes noise by adaptively smoothing the reads from neighboring bases (see Supplementary Methods for details on how reads are combined). In an optional step, reads from multiple experiments, whether replicates or time-series data, are integrated and collectively smoothed using the same Gaussian process framework, which serves to maximize consistent signal while minimizing stochastic noise.

In the final step, PIQ identifies binding sites of each TF in each experiment by iteratively combining direct evidence of binding with indirect analysis of whether the observed DNase-seq data are consistent with a computer-generated model of DNase I hypersensitivity that includes that binding event. First, PIQ preliminarily assigns genomic binding events for each TF motif on the basis of whether a profile exists at each putative binding site. Then, PIQ uses TFsignature profile shapes and magnitudes for each TF to build a model of the expected genomic DNase I hypersensitivity given the assigned binding events. These TF binding estimation and DNase I hypersensitivity model building steps are iteratively performed using a fast approximate machine-learning method called expectation propagation ${ }^{20}$ to arrive at the final binding calls for each motif. PIQ is implemented on the Amazon EC2 cloud server, exploiting parallel computation to substantially speed up run time (Supplementary Methods). Postprocessing to cull motifs whose profiles are indistinguishable from noise (Supplementary Methods) and merging sets of motifs with $>90 \%$ overlapping binding sites reduced the number of informative TF motifs in the cell types we examined in this work to 733.

\section{Benchmarking PIQ}

We applied PIQ, as well as two published DNase-seq-based TF binding detection methods, digital genomic footprinting (DGF; which uses only DNase-seq data) ${ }^{15}$ and Centipede (which, like PIQ, incorporates DNase-seq and motif data) ${ }^{14}$, to published DNase-seq data from K562 cells and validated these predictions against 303 matched ChIP-seq experiments ${ }^{15}$ (Supplementary Table 1 and Supplementary Methods). Compared with other methods, PIQ exhibited higher accuracy in the prediction of sequence-specific TF binding events, as determined by ChIP-seq peaks covering factor motifs, while displaying comparable overall coverage of all ChIP-seq peaks (Supplementary Fig. 2 and Supplementary Table 1).

To summarize these accuracy numbers, we used a standard statistical technique to gauge predictive accuracy, the area under the receiver operating characteristic curve (AUC; Supplementary Methods), which represents the probability of correctly ranking, from ChIPseq data, a bound motif above an unbound motif for each method. Corresponding AUC scores revealed that the predictions of PIQ were more accurate than those of both other methods at every one of the 303 ChIP-seq experiments (PIQ mean $\mathrm{AUC}=0.93$, Centipede mean AUC of 0.87 and DGF mean AUC of 0.65; Fig. 2a and Supplementary Table 1). A similar comparison on six mouse embryonic stem cell ChIP-seq profiles ${ }^{21}$ that matched known motifs also found PIQ to be highly concordant $($ AUC minimum $=0.86$, mean $=0.92$; Fig. 2 b) . The median fraction of total ChIP-seq binding sites recapitulated by PIQ predictions was $66 \%$ for 200 of 303 sequence-specific ChIP-seq experiments with more than half of their sites backed by motifs, and $50 \%$ over all 303 experiments (Supplementary Table 1 and Supplementary Fig. 2). Similarly, median positive predicting value (PPV; Supplementary Methods) scores, which reveal the precision of PIQ predictions over the top 500 predictions, were $76 \%$ for the top quarter of ChIP-seq experiments, $32 \%$ for the 200 motif-enriched experiments noted above and 39.4\% over 194 experiments for which any DNase-seq method achieved $>0 \%$ PPV, substantially outperforming Centipede and DGF. Thus, PIQ was consistently highly concordant with ChIP-seq (median AUC $=0.93$ over 303 ChIP-seq comparison data sets) and thus is a highly accurate tool to uncover TF-DNA binding.

The high correspondence of PIQ output with ChIP-seq results suggests that PIQ is a valuable tool for predicting protein regulatory interactions for hundreds of TFs genome wide. PIQ allows TF binding site prediction with similar accuracy to ChIP-seq for motif-supported direct protein-DNA binding events, with a median AUC of 0.93 . With a small number of replicate experiments PIQ can predict the binding of over 733 factors (Supplementary Methods) and can do so in the absence of specific TF antibodies or tagged TFs. However, PIQ cannot 
Figure 2 Benchmarking PIQ. (a) AUC values (the probability of correctly ranking a bound TF site above an unbound one) for a comparison of PIQ versus ChIP-seq data (PIQ AUC, $x$ axis) and DGF or Centipede versus ChIP-seq data (higher AUC value of DGF or Centipede for each experiment, $y$ axis) for 303 matched ChIP-seq experiments in K562 cells. (b) ROC curves (which show the tradeoff between true positives to false positives as the cutoff for defining what is bound is varied) comparing $\mathrm{mESC}$ stage PIQ binding calls for the TFs Ctcf, c-Myc and Esrrb against matched ChIP-seq binding calls. To calculate ROC curves, we ranked all above-threshold genomic motif instances for each TF according to their PWM motif strength (PWM alone), total adjacent DNase I hypersensitivity in a 400-bp window (DNase HS alone) or the per-site binding score given by PIQ. True positives are compared to false positives at progressively lower ranked sites. Inset, average, minimum and maximum AUC values for six mESC-stage PIQ versus ChIP-seq comparisons.

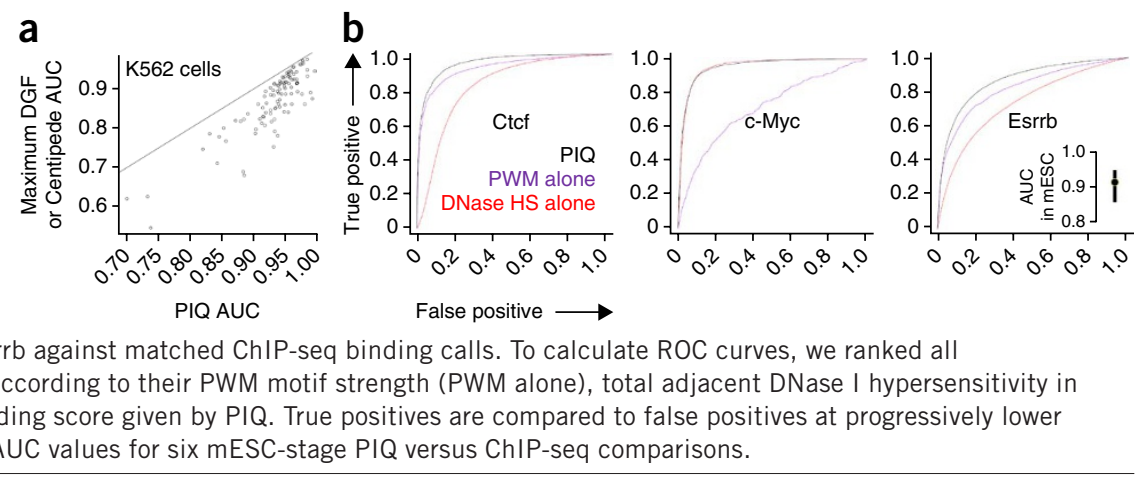

detect TF motif-free binding events that are observed in ChIP-seq data for certain TFs. Some motif-free ChIP-seq events may be mediated by cofactor proteins with diverse sequence specificities, and PIQ would miss these regulatory interactions, although some motif-free events may also be artifacts.

\section{PIQ identifies pioneer transcription factors}

We next used PIQ to explore why ChIP-seq experiments have consistently shown that transcription factors bind to fewer than $5 \%$ of their 5-15-bp thermodynamic high-affinity genomic motifs ${ }^{22,23}$. To explain this disparity, we sought to test the hypothesis that TFs, rather than interacting with the epigenetic environment uniformly, act hierarchically, with some TFs actively manipulating chromatin state and others passively responding to local chromatin architecture. The idea that a subset of TFs, defined as pioneer factors, occupy previously closed chromatin and, once bound, allow other TFs to bind nearby has been proposed previously ${ }^{24-26}$ but not systematically explored. We decided to test whether PIQ, which directly models TF-dependent chromatin accessibility, could discover pioneer factors de novo and characterize TFs into classes based upon their behavior with respect to chromatin accessibility.

We applied PIQ to data from a developmental lineage model that involves the stepwise differentiation of mouse embryonic stem cells (mESCs) to prepancreatic and intestinal endoderm ${ }^{27}$. We induced differentiation of prepancreatic and intestinal endoderm by subjecting mESCs for $6 \mathrm{~d}$ to an in vitro growth factor and small molecule treatment protocol (Fig. 3a). We collected DNase-seq data at two intermediate stages along this stepwise differentiation pathway, mesendoderm (day 3) and endoderm (day 5) as well as from lateral plate mesoderm, which we derived by treating mesendoderm cells with distinct growth factors. This experimental structure yielded a total of six cell states (Fig. 3a) all of which were generated with $>90 \%$ efficiency (Supplementary Fig. 3), providing relatively homogenous populations. We found that PIQ identified extensive changes in TF occupancy through differentiation. TFs most strongly expressed in the mESC state such as Pou5f1, Sox 2 and Esrrb also bound most often in mESCs, and likewise for mesendoderm-enriched TFs Eomes and Irf1, and prepancreatic endoderm-enriched TFs Sox17, Foxa2 and Hoxa1 (Fig. 3b).

We asked whether PIQ could provide an initial understanding of the rules governing the choice of TF binding site. We focused first on whether some TFs act as 'pioneers' 24 , shaping the chromatin landscape and the binding of other TFs. Several reports of TFs possessing pioneer activity exist in the literature $24,26,28-33$, but these reports are empirical experimental studies that do not use standard criteria to define pioneer TF activity and are often unconfirmed functionally. To date to our knowledge no systematic attempts have been taken to categorize pioneer TFs. Although pioneer TFs have been defined in various ways, we probed the existence of pioneer TFs capable of binding to closed chromatin and opening nearby chromatin for future occupancy by other TFs. Using our time series, we designed a pioneer index to measure the expected motif-specific local increase in DNase I accessibility with respect to baseline at sites whose binding changes between successive time points according to PIQ for each of our 733 motifs (Supplementary Methods). A larger pioneer index corresponds to an increase in chromatin opening activity from one time point to the next in our developmental time course.

We found that most motifs showed little appreciable pioneer activity, whereas a small number of motifs open chromatin substantially upon binding (Fig. 3c and Supplementary Table 2). Although there was no clear division between weak pioneers and nonpioneers, a stringent pioneer-index cutoff gave an estimate that 120 of the 733 motifs (16\%) showed pioneer activity, and the motifs with strongest pioneer activity could be classified into ten TF families (Klf/Sp, NFYA, Nrf, ETS, Creb/ATF, Zfp161, KAISO, zinc finger, E2F and CTCF; Supplementary Tables 3 and 4). Of note, previously identified pioneer TFs in the GATA ${ }^{28}, \mathrm{Klf}^{26}$ and $\mathrm{NFYA}^{29}$ families displayed high pioneer indices, whereas FoxA1 (ref. 28), the first identified pioneer, had a low pioneer index.

As binding sites that vary across our observations do not represent a majority of all binding events and are influenced by dynamic TF expression profiles in the particular cell types analyzed, we devised a second metric, the chromatin opening index, to measure the expected static local increase in DNase I accessibility attributed to each motif (Supplementary Methods). The chromatin opening index is highly concordant with the pioneer index $\left(r^{2}=0.98\right.$, Fig. 3d, Supplementary Fig. 4 and Supplementary Table 2), indicating that pioneers can be identified through their static association with open chromatin, thus providing an alternative metric for pioneer TFs that does not require temporal DNase-seq data. TF families with high chromatin opening index scores are conserved in K562 cells $\left(r^{2}=0.84\right.$, Supplementary Fig. 4), indicating that chromatin opening is a TF-intrinsic activity consistent across cell type and species.

To determine whether pioneer motifs facilitate binding of other TFs in addition to governing chromatin structure, we devised the social index, the mean number of PIQ-identified binding sites within 200 bp of PIQ-called binding events for a given TF (Supplementary Methods) and found that pioneer TFs in most cases had more neighbors than nonpioneer TFs (Fig. 3e and Supplementary Table 2). In all analyses, we excluded sites adjacent to annotated transcription start sites to avoid artifacts associated with the strong nucleosome depletion at promoters ${ }^{15,16}$, and the results remained consistent after a more stringent removal of unannotated promoters detected through global run-on sequencing, RNA sequencing and by using histone marks characteristic of promoters (Supplementary Fig. 4). 

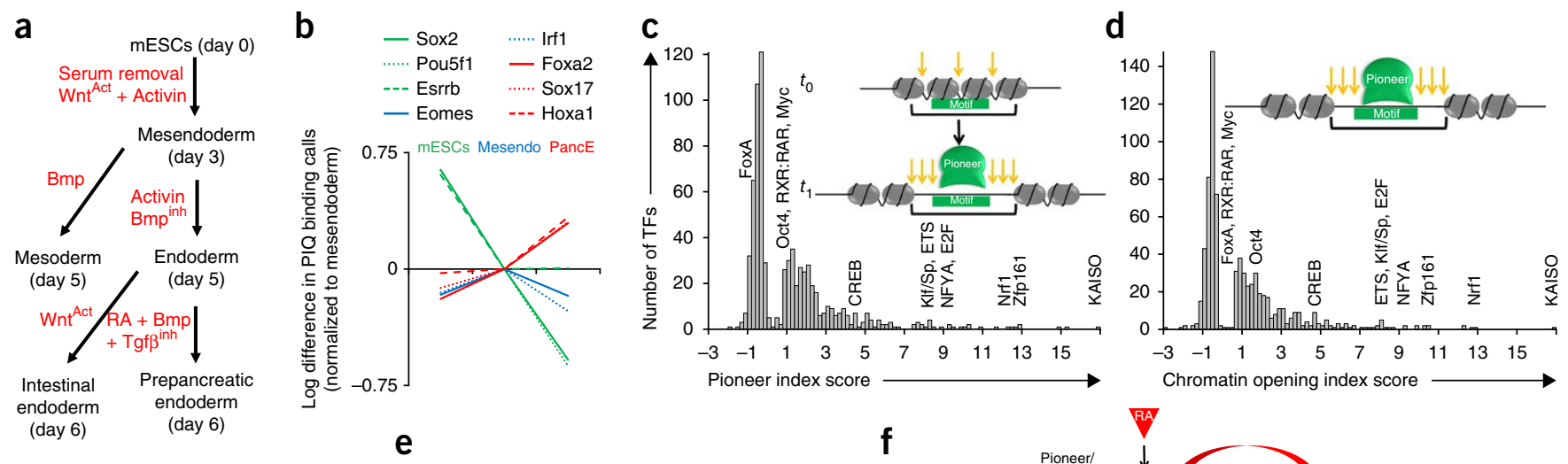

Figure 3 Systematic identification of pioneer TFs. (a) Outline of mESC-derived populations used for dynamic DNase-seq analysis. Wnt ${ }^{A c t}$, activation of Wnt; Bmpinh, inhibition of Bmp; Tgf $\beta^{\text {inh }}$, inhibition of Tgf $\beta$. (b) Differences in PIQ-detected binding sites for eight selected TFs with strong microarray expression values in mESCs (green), mesendoderm (Mesendo, blue) and prepancreatic endoderm (PancE, red). For each TF at each stage, PIQ calculates a score representing the overall number and strength of binding sites, plotted in natural log PIQ binding strength units normalized to mesendoderm
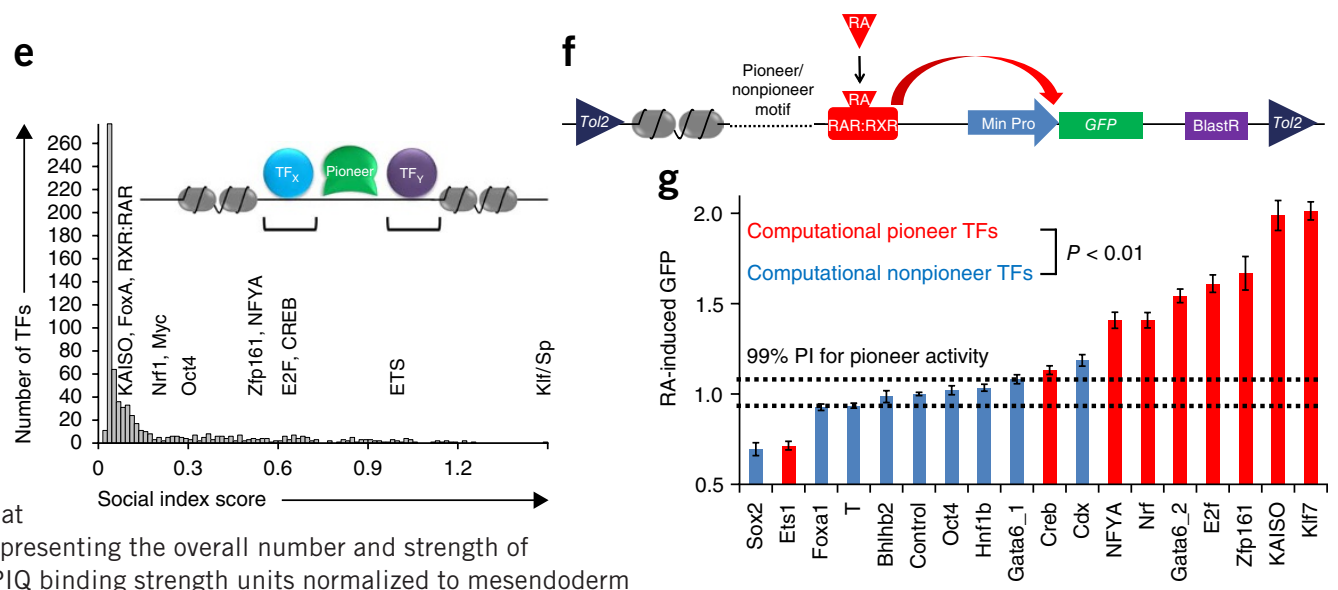
values. (c) Pioneer index log odds scores for all PIQ motifs. (d) Chromatin opening index log odds scores for all PIQ motifs. Yellow arrows represent DNase I accessibility. (e) Social index scores for all PIQ motifs. Scores of selected pioneer and nonpioneer TFs in a-c are noted. (f) Schematic of modular Tol2 transposon-based pioneer reporter system to test pioneer and nonpioneer motifs for chromatin-opening ability. Chromatin openness is read out by the level of RA-induced RAR:RXR DNA binding and consequent GFP transcriptional activation, as measured by flow cytometric fluorescence. (g) Average increase in flow cytometric fluorescence after RA addition for 18 pioneer reporter lines grouped as predicted pioneer and nonpioneer TFs, normalized to RA-induced GFP of the control reporter line. Error bars, s.e.m. Dotted line represents $99 \%$ prediction interval (PI) based on control RA-induced GFP fluorescence, indicating lines with RA-induced GFP expression out of the predicted control range. $n=4$ experiments, $P<0.01, t$-test.

We experimentally tested the ability of a variety of predicted pioneer and control motifs to open up surrounding chromatin and allow other TFs to bind. To evaluate these criteria in a high-throughput, functional assay, we designed 18 versions of a reporter vector driven by a strong retinoid $\mathrm{X}$ receptor:retinoic acid receptor (RXR:RAR) motif directly adjacent to a pioneer or nonpioneer motif at a locus $>1$ kilobase $(\mathrm{kb})$ from a minimal promoter and GFP reporter gene (Fig. 3f). We chose the RXR:RAR motif for three reasons. First, RXR:RAR binding showed no effect on surrounding chromatin in a computational analysis (Supplementary Table 2). Second, nuclear hormone receptors, which bind the RXR:RAR motif, respond primarily to surrounding chromatin state rather than specific cofactor interactions ${ }^{34}$ (see below). Third, the RXR:RAR motif allows strong inducible expression of GFP upon addition of retinoic acid (RA), allowing a straightforward quantitative readout of cellular fluorescence intensity. We inserted this vector into the genome of mESCs by means of Tol2 transposition ${ }^{35}$ followed by antibiotic selection, which enabled random genomic integration in a highly polyclonal fashion (>1,000 distinct clones per reporter line), thus controlling for site-specific effects. Consistent with this idea, biological replicates of several lines produced from distinct rounds of Tol 2 transposition yielded highly reproducible results (Supplementary Fig. 5). We then used flow cytometry to measure cellular GFP levels in mESCs after $24 \mathrm{~h}$ in the presence or absence of RA and interpreted RA-induced increases in GFP fluorescence as a correlate of the accessibility of the RXR:RAR site (Fig. 3g).
The pioneer reporter assay data support the computational pioneer TF predictions. Eight of nine predicted pioneer motifs showed significantly above control RA-induced GFP fluorescence as compared with only one of eight nonpioneer motifs (Fig. 3g), and pioneer TFs on average promoted significantly higher RA-induced GFP than did controls $(P<0.01, t$-test $)$. None of the 18 tested motifs showed significant GFP induction $(P<0.01, t$-test $)$ in the absence of RA as compared to the control line (Supplementary Fig. 5), indicating that pioneer and nonpioneer motifs alike did not activate gene expression significantly on their own. Quantitative RT-PCR (RT-qPCR) analyses also confirmed that RA-induced transcripts did not span the promoter region and pioneer sequences still increased RA-induced GFP expression when the enhancer was $3 \mathrm{~kb}$ away from the minimal promoter, confirming that the reporter constructs acted as distal enhancers (Supplementary Fig. 5). Last, to control for the relative expression of TFs, we performed the reporter assays in mesendoderm and in the presence of ectopically expressed pioneer and nonpioneer TFs, obtaining consistent results (Supplementary Fig. 5).

Asymmetrical opening of chromatin by directional pioneer TFs Evidence exists that TFs deposit histone marks asymmetrically ${ }^{36}$. We identified a subset of pioneer TF families that open chromatin more strongly on one side of their motif than on the other (Fig. 4a and Supplementary Fig. 6). We refer to factors that possess this asymmetrical chromatin opening ability as 'directional pioneers.' To quantify activity of directional pioneers, we measured the expected 
Figure 4 Asymmetrical chromatin opening by directional pioneers. (a) Per-base chromatin opening index log odds scores, which represent expected local increase in hypersensitivity induced by TF binding at all above-threshold genomic motifs for Creb1, KIf7, NFYA and Zfp161. $x$ axis for each plot is \pm 200 bp from the motif center. (b) Experimental validation of directional pioneers. Average increase in flow cytometric fluorescence after RA addition for pioneer reporter lines for the indicated motifs. RC (reverse complement) and Fw (forward) show reporter results when the motif orientation was such that the RAR site was on the left or right, respectively, of the motif with respect to the data in a. All plots are normalized to control line RA-induced GFP fluorescence as in Figure 3f. Error bars, s.e.m., and a 99\% prediction interval (PI) is shown as in Figure $3 f$.

difference in chromatin opening on either side of each pioneer motif (Supplementary Table 3) and identified strong directional pioneer activity in the Klf/Sp, NFYA, Creb/ATF and Zfp161 pioneer TF families. As we cannot observe directional pioneer activity at palindromic motifs because PIQ cannot orient them, we note that the directional pioneer TF Creb/ATF has multiple PWMs, one of which is nonpalindromic. Although directional motifs are known to be important at promoters ${ }^{37}$, our analyses excluded regions adjacent to transcription start sites, and we did not find appreciable transcript production or promoter-characteristic histone marks at distal pioneer sites (Supplementary Fig. 4). Thus, the unidirectional opening of chromatin relative to pioneer TF motif represents a property of certain TFs that to our knowledge has not been described.

To experimentally assess directional pioneer activity, we performed reporter analysis on four motifs displaying strongly directional pioneer activity (Fig. 4b), placing both motif orientations relative to the RXR:RAR site. In all four cases, RA-induced GFP was significantly $(P<0.01, t$-test $)$ stronger in the direction predicted to have higher pioneer activity (Fig. 4b), and as predicted, NFYA, Creb and Zfp161 only opened chromatin in a single direction from their motif. Directional pioneer activity did not occur during transient

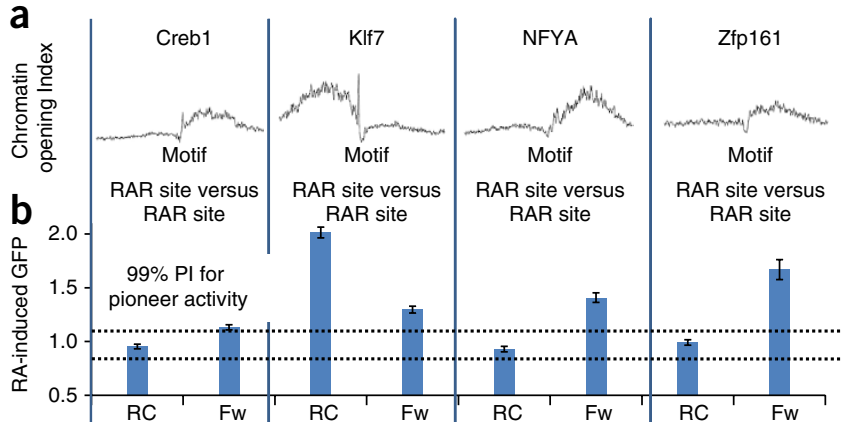

transfection (Supplementary Fig. 5), suggesting that this activity occurs through interaction with the local chromatin state.

\section{Settler TFs depend on open chromatin for binding}

Next we reasoned that classifying TFs by their interactions with chromatin might reveal distinctions in how TFs choose binding sites. As pioneers have been shown to scan nucleosomal DNA for their motifs ${ }^{38}$, we reasoned that they may be more likely than other TFs to bind to their motif wherever it occurs. To assess this idea, we devised a metric to indicate the likelihood of a TF binding an instance of its motif, the correlation of PWM score and binding probability (referred to hereafter as 'motif dependence'). Plotting motif dependence against the chromatin opening index, we found a significant $(P<0.01, t$-test $)$ but imperfect positive correlation between motif dependence and chromatin opening (Fig. 5a and Supplementary Table 4), suggesting that pioneer TFs generally do not bind to a high fraction of their genomic motif candidates. Several nonpioneer TFs, including REST, also displayed strong motif dependence (Fig. 5a and Supplementary Table 4). Motif dependence was uncorrelated with motif information content, suggesting that it is not an artifact of database PWM quality (Supplementary Fig. 7). Thus, although pioneers TFs are more likely
Figure 5 Binding of settler TFs is governed by underlying chromatin state. (a) Motif dependence versus chromatin opening index for all 733 motifs in mouse lineage. Selected TFs are labeled, and the linear trendline shows imperfect but significant positive correlation ( $F$ test). (b) Chromatin dependence versus chromatin opening index for all 733 motifs in mouse lineage. Classes of pioneer TFs, settler TFs and migrant TFs as defined by their chromatin opening and dependence properties are shaded, and selected members of each class are listed. (c) K562 cell DNase-seq chromatin openness score versus binned K562 ChIP-seq binding probability at strong motifs for Elf1 (ETS family, pioneer), c-Myc (settler) and the average of all ChIP-seq experiments. (d) Contour plots show log odds binding probability (contour) for bins of strong motifs at varying chromatin openness scores and PWM scores for the K562 cell ChIP-seq TF clusters displaying chromatin dependence only (top) or combinatorial motif dependence and chromatin dependence (bottom). (e) Change in number of true positive PIQ calls per TF motif at a $10 \%$ false discovery rate as a result of incorporating motif dependence and chromatin dependence as prior information for all K562 cell ChIP-seq motif comparisons. Prior information improved PIQ accuracy for most TFs. a

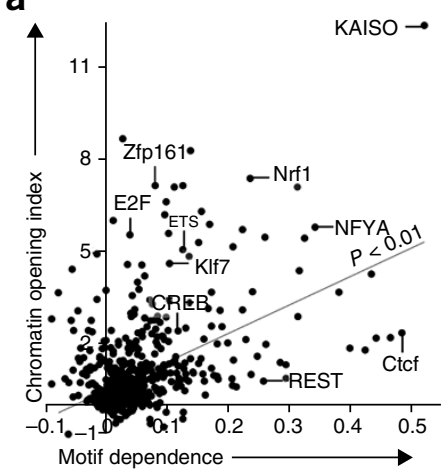

C

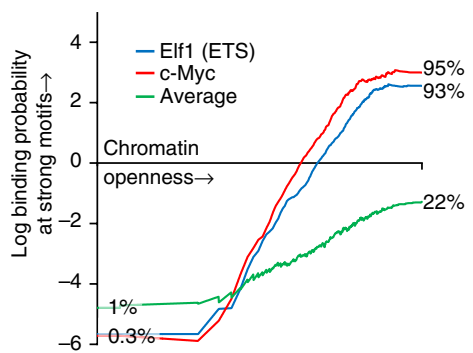

b

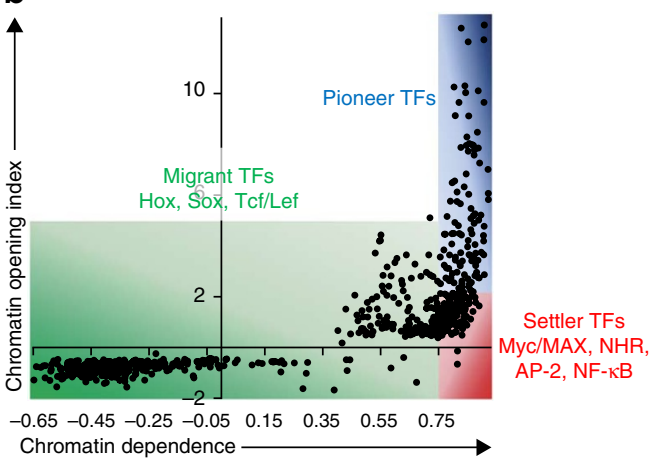

d Chromatin dependent only $\mathbf{e}$
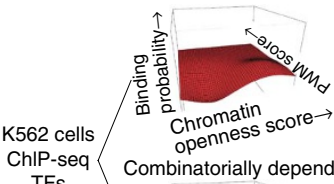
Chromatin score $\rightarrow$ TFs
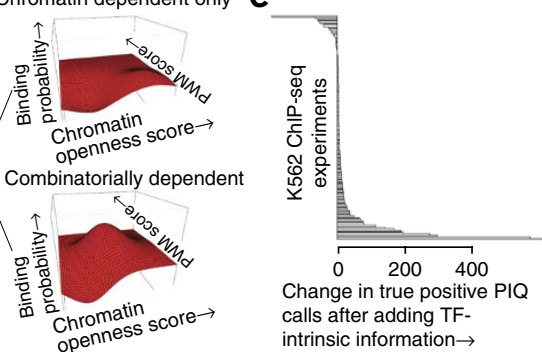

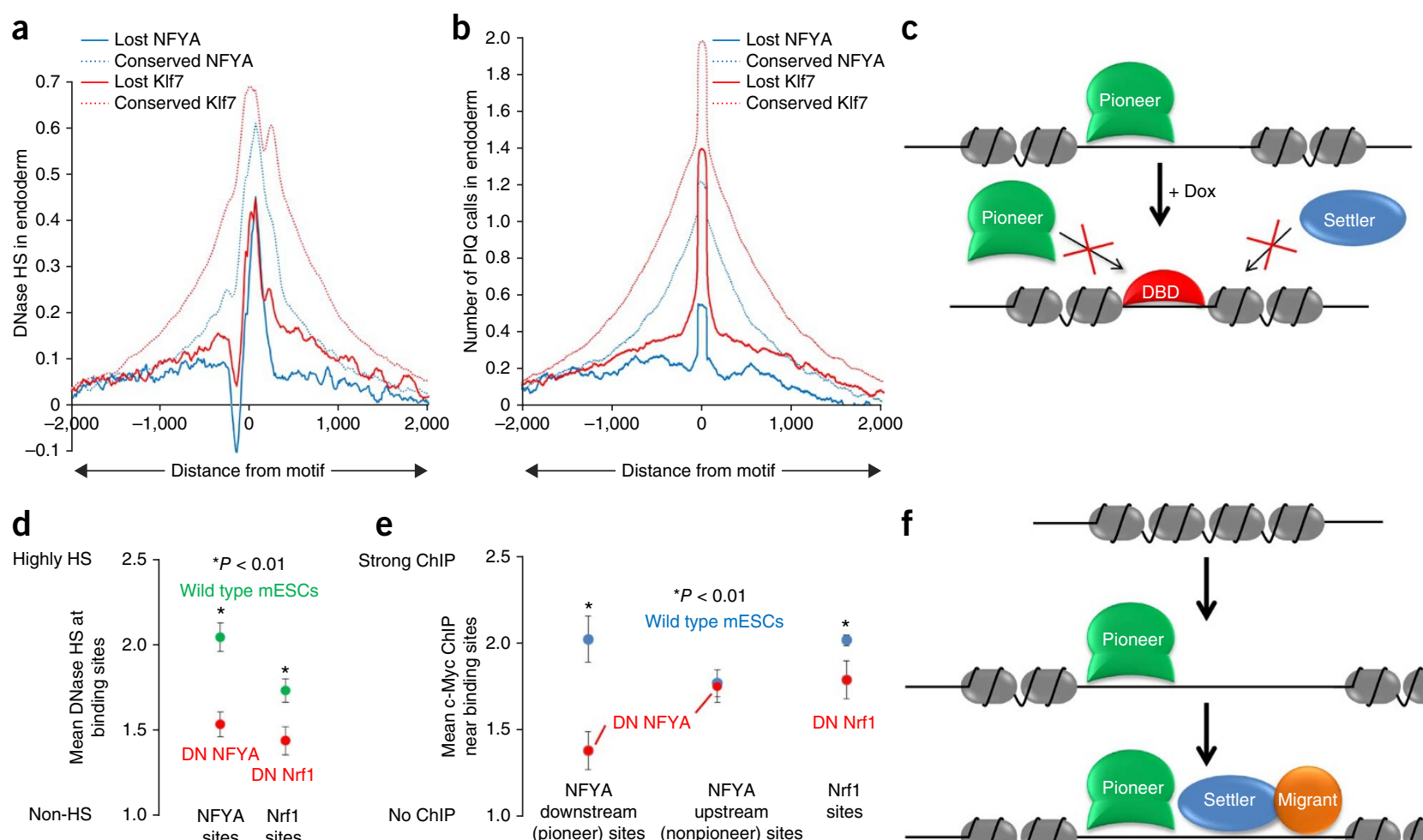

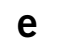

Strong ChIP

Figure 6 Pioneer TFs control chromatin state and settler TF binding. (a,b) Per-base average DNase I hypersensitivity (HS) (a) and number of PIQ binding sites (b) within $4 \mathrm{~kb}$ of KIf7 and NFYA motifs for sites conserved or lost between mesendoderm and endoderm stages. (c) Schematic of pioneer dominant negative (DN) competition experiments in which doxycycline (Dox) induces DN allele-encoded pioneer TF expression (DBD), which should block pioneer-induced chromatin opening and prevent settler binding to opened chromatin. (d) Mean DNase I hypersensitivity at several strong binding sites for NFYA and Nrf1 in wild-type (WT) (green) or double-negative allele-encoded NFYA or Nrf1 (DN NFYA, DN Nrf1) mESCs, normalized to background DNase I activity at non-hypersensitive sites. ${ }^{*} P<0.01$ between average DNase I HS between WT and DN cells using $t$-test ( $n=4$ experiments). (e) Mean ChIP enrichment for four c-Myc sites downstream (in direction of predicted pioneer activity) of NFYA (left), upstream (in direction of predicted nonpioneer activity) of NFYA (middle) or adjacent to Nrf1 (right) in WT or DN NFYA, DN Nrf1 mESCs, normalized to positive and negative control genomic c-Myc sites. ${ }^{*} P<0.01, t$-test ( $n=3$ experiments). Error bars (d,e), s.e.m. (f) Model of TF binding hierarchy. Pioneer TFs open chromatin, some directionally, and open chromatin is populated by settler TFs and by certain combinations of migrant TFs.

to bind their motifs than are nonpioneers, they still rely on facets other than their motif in a majority of their binding decisions.

Among nonpioneer TFs, we reasoned that some TFs might be disproportionately dependent on the preexisting chromatin state as established by pioneer TFs. We explored this possibility computationally by measuring the correlation between DNase I accessibility surrounding high-confidence TF motifs and binding probability (Supplementary Table 4). Plotting this chromatin-dependence metric against the chromatin opening index, which controls for TF-intrinsic chromatin opening, we found that TFs vary substantially in their dependence on chromatin openness in order to bind genomic DNA (Fig. 5b). A subset of TFs were highly likely to bind wherever their motif occurred in an open chromatin landscape but did not open chromatin themselves.

We coin the term 'settler' TFs to define the set of TFs whose binding is predominantly dependent on the openness of chromatin at their motifs. Chromatin dependence of TFs was graded, but a stringent cutoff in the chromatin-dependence metric gave an estimate that 131 of the 733 motifs (18\%) act as settler TFs (Supplementary Table 4). The majority of nonpioneer TFs, which we term 'migrant' TFs, bind only sporadically even when chromatin at their motifs is open and are presumably more heavily dependent on specific cofactor interactions (see Supplementary Table $\mathbf{4}$ for factor-specific classifications in the mESC pancreatic lineage). Accurate a priori prediction (AUC $>0.9)$ of
ChIP-seq genomic binding of 'settler' TFs, such as members of the Myc/ MAX, nuclear hormone receptor (i.e., RXR:RAR), Ap-2 and NF- $\mathrm{KB}$ families, can be obtained simply by measuring DNase I accessibility surrounding their motifs (Figs. 2b and 5c), so binding of settler TFs can be accurately determined solely based on chromatin accessibility in the absence of ChIP or DNase I profile information. Pioneer TF binding can also be predicted a priori by local DNase I accessibility (Fig. 5c), presumably a result of pioneer-induced chromatin opening at binding sites either in the profiled developmental stage or at a prior time point. Thus, we have identified a class of settler TFs, which to our knowledge has not been described, that obey one simple rule, binding DNA when chromatin is open, establishing settler TFs as a class whose binding is directly dependent on the chromatin-opening ability of pioneer TFs.

Although pioneer TFs and settler TFs typify chromatin opening and chromatin dependence, respectively, we reasoned that the motifdependence properties and chromatin-dependence properties of migrant TFs might also contribute to their binding decisions. To test this hypothesis, we clustered TFs possessing matched ChIP-seq and DNase-seq experiments in K562 cells ${ }^{39}$ by their combination of motif dependence and chromatin dependence. We found that TFs broadly fell into two categories: those for which ChIP-seq binding probability increases only with chromatin openness and those for which binding probability is combinatorially linked to motif score and chromatin openness (Fig. 5d and Supplementary Fig. 7). Modifying PIQ to 
incorporate these TF-intrinsic binding dependencies into its binding calls improves predictive accuracy for a majority of TFs with matched ChIP-seq data (Fig. 5e), indicating that TF-intrinsic chromatin interaction can be exploited to improve binding prediction. Although we have not included data on histone modification or DNA methylation status in PIQ, we found that DNase I hypersensitive regions and PIQidentified TF binding sites have low levels of DNA methylation in mESCs (Supplementary Fig. 7). This suggests that future addition of data types may further improve binding prediction.

\section{Hierarchical binding of pioneer and settler TFs}

Our hierarchical binding model predicts that loss of pioneer TF binding should result in closing of chromatin and loss of settler TF binding, at times directionally. Sites at which pioneer TF binding is lost during mESC differentiation do in fact show dramatic loss of DNase I hypersensitivity and of adjacent TF binding (Fig. 6a,b). To address this idea mechanistically, we constructed mESCs with doxycyclineinducible dominant negative alleles for two pioneer TFs, NFYA and Nrf1, that consist solely of DNA-binding domains (Fig. 6c). These proteins encoded by dominant negative alleles should bind to their cognate motifs and compete with their native counterparts, blocking pioneer TF-induced increase in chromatin accessibility. Creation of doxycycline-inducible lines avoids the lethality associated with knockouts of these TFs ${ }^{40,41}$. DNase I hypersensitivity analysis followed by quantitative PCR (DNase-qPCR) analysis at a set of strongly bound sites revealed that both dominant negative allelele-encoded NFYA and Nrf1 significantly reduced hypersensitivity at their respective binding sites (Fig. 6d). Furthermore, impairing NFYA and Nrf1 binding also impaired adjacent binding of the settler TF c-Myc at several genomic loci (Fig. 6e). Consistent with our prediction of NFYA's directional pioneer activity (Fig. 4), impairing NFYA binding diminished c-Myc binding when the c-Myc site was downstream of the NFYA site but not upstream of it (Fig. 6e). Thus, pioneer TF binding is required to maintain open chromatin and to allow nearby settler TF binding, confirming that pioneer TFs sit atop a TF binding hierarchy.

\section{DISCUSSION}

We conclude that PIQ offers a window into TF binding and behavior and has facilitated the elucidation of pioneer TFs that represent a mechanistically diverse set of TFs that have a disproportionately large role in organizing chromatin structure. In a chromatin-based view of TF binding, pioneer TFs shape the chromatin landscape, allowing settler TFs and specific combinations of migrant TFs to populate open chromatin (Fig. 6f). We have shown both computationally and experimentally that through mESC differentiation, gain of pioneer TF binding opens chromatin and that loss of pioneer TF binding closes chromatin, and so we posit that pioneer TFs have an important role in controlling the TF binding dynamics that control acquisition of cell fate.

We designed PIQ to model factors that directly modulate chromatin accessibility, and PIQ is thus uniquely capable of identifying pioneer factors from DNase-seq experiments. PIQ fits a background read model over the entire genome, which allows us to precisely quantify how much a transcription factor opens chromatin relative to both other factors and genomic background. Prior methods such as Centipede model TF binding on a factor-to-factor basis and therefore would normalize out cross-factor effects. In addition, the chromatinopening index is a natural extension of a TF's profile in PIQ, whereas in DGF or Centipede profiles are by definition normalized to a mean of zero and do not indicate chromatin opening. We have found in practice that this more detailed model of chromatin accessibility has made it possible to detect TFs with indistinct footprints but large chromatin effects. In some of our identified pioneers such as Gata6, PIQ detects distinct binding sites whereas Centipede fails to do so (Supplementary Fig. 8).

Recent work ${ }^{42,43}$ has suggested that DNase I sequence bias may add noise to narrow DNase-seq footprints. In PIQ, TF binding detection is performed on a TF-specific profile, extending $400 \mathrm{bp}$ from each motif and thus is not limited to the 5-10-bp footprint itself (Supplementary Fig. 9). PIQ performs a profile-level significance test for whether or not an estimated TF profile is significant outside its motif match region, and all identified pioneer TFs are highly significant (Supplementary Fig. 9).

Our identification of pioneer and settler TFs is limited by the breadth of the motifs used in PIQ, by the extent of expression and dynamic binding of TFs in the cell types analyzed in this data set, and by the focus on single motifs, which may exclude emergent chromatin opening of TF combinations. Thus the list of pioneer and settler TF families should expand with the collection of more DNase-seq data and TF motifs ${ }^{15}$. We further note that TFs that do not open chromatin but still facilitate the binding of other factors and those that induce chromatin repression are not captured by our DNase I-based assay. Notably, the most well-studied pioneer TF, Foxa1, had a relatively low score in all indices (Fig. 3c-e). This may result from the dual role of Foxal as a chromatin-opening and chromatin-compacting agent ${ }^{44,45}$, its dependence on prior binding of Foxd3 (ref. 46) whose strong expression in mESCs could obscure its pioneer activity in this lineage, or its minimal role in coordinating chromatin structure as determined by knockout studies in mouse liver ${ }^{47}$. In any case, this result exemplifies that the computational approach taken here focuses on pioneer TFs that increase DNase I hypersensitivity when they bind and thus does not exhaustively identify pioneer TFs.

Comparing mechanisms by which pioneer TFs function will be a fertile area for future research. Codifying TF properties is a step on the road to a priori prediction of TF binding and gene-network modeling. And as recent work has implicated pioneer TFs in cellular reprogramming ${ }^{26}$, categorizing pioneer and settler TFs could lead to principled manipulation of cell fate.

PIQ implementation and data are available at http://piq.csail.mit. edu/ and as Supplementary Data.

\section{METHODS}

Methods and any associated references are available in the online version of the paper.

\section{Accession codes. Gene Expression Omnibus: GSE53776.}

Note: Any Supplementary Information and Source Data files are available in the online version of the paper.

\section{ACKNOWLEDGMENTS}

We thank G. Losyev, J. Huynh and members of the Massachusetts Institute of Technology BioMicro Center for technical assistance, K. Kawakami (National Institute of Genetics) for Tol2 plasmids, H. Wichterle (Columbia University) for mESCs, and R. Maas for help with the manuscript. We acknowledge funding from the US National Institutes of Health Common Fund 5UL1DE019581, RL1DE019021 and 5TL1EB008540; the Harvard Stem Cell Institute's Sternlicht Director's Fund award to R.I.S., and NIH grants 1U01HG007037 and 5P01NS055923 to D.K.G.

\section{AUTHOR CONTRIBUTIONS}

Experiments were designed by R.I.S., T.H., C.W.O. and D.K.G. DNase-seq experiments were conducted by R.I.S., S.L., A.A.B. and J.P.v.H.,

reporter experiments were conducted by R.I.S. and V.K., and dominant negative experiments were conducted by R.I.S. and A.A.B. PIQ was designed and implemented by T.H. and C.W.O., DNase-seq and PIQ computational analyses 
were performed by T.H., C.W.O., D.K.G. and T.J. The manuscript was prepared by R.I.S., T.H., C.W.O. and D.K.G.

\section{COMPETING FINANCIAL INTERESTS}

The authors declare no competing financial interests.

Reprints and permissions information is available online at http://www.nature.com/ reprints/index.html.

1. Takahashi, K. \& Yamanaka, S. Induction of pluripotent stem cells from mouse embryonic and adult fibroblast cultures by defined factors. Cell 126, 663-676 (2006).

2. Hanna, J.H., Saha, K. \& Jaenisch, R. Pluripotency and cellular reprogramming: facts, hypotheses, unresolved issues. Cell 143, 508-525 (2010).

3. Mullen, A.C. et al. Master transcription factors determine cell-type-specific responses to TGF-beta signaling. Cell 147, 565-576 (2011).

4. Trompouki, E. et al. Lineage regulators direct BMP and Wnt pathways to cell-specific programs during differentiation and regeneration. Cell 147, 577-589 (2011).

5. Young, R.A. Control of the embryonic stem cell state. Cell 144, 940-954 (2011).

6. Davidson, E.H. Emerging properties of animal gene regulatory networks. Nature 468, 911-920 (2010).

7. Johnson, D.S., Mortazavi, A., Myers, R.M. \& Wold, B. Genome-wide mapping of in vivo protein-DNA interactions. Science 316, 1497-1502 (2007).

8. Guo, Y. et al. Discovering homotypic binding events at high spatial resolution. Bioinformatics 26, 3028-3034 (2010).

9. Guo, Y., Mahony, S. \& Gifford, D.K. High resolution genome wide binding event finding and motif discovery reveals transcription factor spatial binding constraints. PLoS Comput. Biol. 8, e1002638 (2012).

10. Boyle, A.P. et al. High-resolution mapping and characterization of open chromatin across the genome. Cell 132, 311-322 (2008).

11. Weintraub, H. \& Groudine, M. Chromosomal subunits in active genes have an altered conformation. Science 193, 848-856 (1976).

12. Wu, $\mathrm{C}$. The $5^{\prime}$ ends of Drosophila heat shock genes in chromatin are hypersensitive to DNase I. Nature 286, 854-860 (1980).

13. Boyle, A.P. et al. High-resolution genome-wide in vivo footprinting of diverse transcription factors in human cells. Genome Res. 21, 456-464 (2011).

14. Pique-Regi, R. et al. Accurate inference of transcription factor binding from DNA sequence and chromatin accessibility data. Genome Res. 21, 447-455 (2011).

15. Neph, S. et al. An expansive human regulatory lexicon encoded in transcription factor footprints. Nature 489, 83-90 (2012).

16. Thurman, R.E. et al. The accessible chromatin landscape of the human genome. Nature 489, 75-82 (2012).

17. Hesselberth, J.R. et al. Global mapping of protein-DNA interactions in vivo by digital genomic footprinting. Nat. Methods 6, 283-289 (2009).

18. Boyle, A.P. et al. High-resolution genome-wide in vivo footprinting of diverse transcription factors in human cells. Genome Res. 21, 456-464 (2011).

19. Chen, X., Hoffman, M.M., Bilmes, J.A., Hesselberth, J.R. \& Noble, W.S. A dynamic Bayesian network for identifying protein-binding footprints from single moleculebased sequencing data. Bioinformatics 26, i334-i342 (2010).

20. Minka, T. Expectation propagation for approximate Bayesian inference. Proceedings of the 17th Conference in Uncertainty in Artificial Intelligence 362-369 (2001).

21 . Chen, X. et al. Integration of external signaling pathways with the core transcriptional network in embryonic stem cells. Cell 133, 1106-1117 (2008).

22. Joseph, R. et al. Integrative model of genomic factors for determining binding site selection by estrogen receptor-alpha. Mol. Syst. Biol. 6, 456 (2010).

23. Kaplan, T. et al. Quantitative models of the mechanisms that control genome-wide patterns of transcription factor binding during early Drosophila development. PLoS Genet. 7, e1001290 (2011).
24. Zaret, K.S. \& Carroll, J.S. Pioneer transcription factors: establishing competence for gene expression. Genes Dev. 25, 2227-2241 (2011).

25. Gualdi, R. et al. Hepatic specification of the gut endoderm in vitro: cell signaling and transcriptional control. Genes Dev. 10, 1670-1682 (1996).

26. Soufi, A., Donahue, G. \& Zaret, K.S. Facilitators and impediments of the pluripotency reprogramming factors' initial engagement with the genome. Cell 151, 994-1004 (2012).

27. Sherwood, R.I., Maehr, R., Mazzoni, E.O. \& Melton, D.A. Wht signaling specifies and patterns intestinal endoderm. Mech. Dev. 128, 387-400 (2011).

28. Cirillo, L.A. et al. Opening of compacted chromatin by early developmental transcription factors HNF3 (FoxA) and GATA-4. Mol. Cell 9, 279-289 (2002).

29. Nardini, M. et al. Sequence-specific transcription factor NF-Y displays histone-like DNA binding and H2B-like ubiquitination. Cell 152, 132-143 (2013).

30. Budry, L. et al. The selector gene Pax7 dictates alternate pituitary cell fates through its pioneer action on chromatin remodeling. Genes Dev. 26, 2299-2310 (2012).

31. Eeckhoute, J., Carroll, J.S., Geistlinger, T.R., Torres-Arzayus, M.I. \& Brown, M. A cell-type-specific transcriptional network required for estrogen regulation of cyclin D1 and cell cycle progression in breast cancer. Genes Dev. 20, 2513-2526 (2006).

32. Hori, S. c-Rel: a pioneer in directing regulatory T-cell lineage commitment? Eur. J. Immunol. 40, 664-667 (2010).

33. Treiber, $\mathrm{T}$. et al. Early $\mathrm{B}$ cell factor 1 regulates $\mathrm{B}$ cell gene networks by activation, repression, and transcription-independent poising of chromatin. Immunity $\mathbf{3 2}$, 714-725 (2010).

34. John, S. et al. Chromatin accessibility pre-determines glucocorticoid receptor binding patterns. Nat. Genet. 43, 264-268 (2011)

35. Kawakami, K. \& Noda, T. Transposition of the Tol2 element, an Ac-like element from the Japanese medaka fish Oryzias latipes, in mouse embryonic stem cells. Genetics 166, 895-899 (2004).

36. Kundaje, A. et al. Ubiquitous heterogeneity and asymmetry of the chromatin environment at regulatory elements. Genome Res. 22, 1735-1747 (2012).

37. Eddy, J. et al. G4 motifs correlate with promoter-proximal transcriptional pausing in human genes. Nucleic Acids Res. 39, 4975-4983 (2011).

38. Sekiya, T., Muthurajan, U.M., Luger, K., Tulin, A.V. \& Zaret, K.S. Nucleosomebinding affinity as a primary determinant of the nuclear mobility of the pioneer transcription factor FoxA. Genes Dev. 23, 804-809 (2009).

39. ENCODE Project Consortium. et al. An integrated encyclopedia of DNA elements in the human genome. Nature 489, 57-74 (2012).

40. Bhattacharya, A. et al. The B subunit of the CCAAT box binding transcription factor complex (CBF/NF-Y) is essential for early mouse development and cell proliferation. Cancer Res. 63, 8167-8172 (2003)

41. Huo, L. \& Scarpulla, R.C. Mitochondrial DNA instability and peri-implantation lethality associated with targeted disruption of nuclear respiratory factor 1 in mice. Mol. Cell. Biol. 21, 644-654 (2001).

42. Koohy, H., Down, T.A. \& Hubbard, T.J. Chromatin accessibility data sets show bias due to sequence specificity of the DNase I enzyme. PLOS ONE 8, e69853 (2013).

43. He, H.H. et al. Refined DNase-seq protocol and data analysis reveals intrinsic bias in transcription factor footprint identification. Nat. Methods doi:10.1038/ nmeth.2762 (8 December 2013).

44. Sekiya, T. \& Zaret, K.S. Repression by Groucho/TLE/Grg proteins: genomic site recruitment generates compacted chromatin in vitro and impairs activator binding in vivo. Mol. Cell 28, 291-303 (2007).

45. Watts, J.A. et al. Study of FoxA pioneer factor at silent genes reveals Rfx-repressed enhancer at $\mathrm{Cdx} 2$ and a potential indicator of esophageal adenocarcinoma development. PLoS Genet. 7, e1002277 (2011).

46. Xu, J. et al. Transcriptional competence and the active marking of tissue-specific enhancers by defined transcription factors in embryonic and induced pluripotent stem cells. Genes Dev. 23, 2824-2838 (2009).

47. Li, Z., Schug, J., Tuteja, G., White, P. \& Kaestner, K.H. The nucleosome map of the mammalian liver. Nat. Struct. Mol. Biol. 18, 742-746 (2011). 


\section{ONLINE METHODS}

Protein interaction quantitation algorithm. Mathematical rationale, principles and implementation of the PIQ algorithm are described in Supplementary Methods.

Mouse embryonic stem cell line generation, culture and differentiation. Mouse embryonic stem cell culture and endoderm differentiation was modified slightly from previously published protocols ${ }^{27}$. Undifferentiated 129P2/OlaHsd mESCs were maintained on gelatin-coated plates with mouse embryonic fibroblast (MEF) feeders in mESC medium composed of Knockout DMEM (Life Technologies) supplemented with 15\% defined FBS (HyClone), $0.1 \mathrm{mM}$ nonessential amino acids (Life Technologies), 1\% Glutamax (Life Technologies), $0.55 \mathrm{mM}$ 2-mercaptoethanol (Sigma) and 1× ESGRO LIF (Millipore).

Before differentiation, ESCs were passaged onto gelatin-coated plates for $25 \mathrm{~min}$ to deplete MEFs. MEF-depleted ESCs were then seeded at $1 \times 10^{4}$ cells $/ \mathrm{cm}^{2}$ onto gelatin-coated dishes in mESC medium. After 12-24 h, medium was changed to Advanced DMEM (Life Technologies) supplemented with N-2 (Life Technologies), B27 without vitamin A (Life Technologies) and 1\% Glutamax. After 44-48 h, medium was changed to Advanced DMEM with 2\% FBS, 1\% Glutamax, $5 \mathrm{nM} \mathrm{GSK-3} \mathrm{inhibitor} \mathrm{XV} \mathrm{and} 50 \mathrm{ng} / \mathrm{ml}$ Escherichia coli-derived Activin A (Peprotech) for $24 \mathrm{~h}$ to produce mesendoderm. For endoderm differentiation, cells were then fed with Advanced DMEM with $2 \%$ FBS, $1 \%$ Glutamax, $50 \mathrm{ng} / \mathrm{ml}$ Activin A and $1 \mu \mathrm{M}$ dorsomorphin (Sigma) for $48 \mathrm{~h}$. For intestinal endoderm differentiation, cells at the endoderm stage were fed for $24 \mathrm{~h}$ with Advanced DMEM with B-27 supplement without vitamin A, 1\% Glutamax and 100 nM GSK-3 inhibitor XV. For differentiation of prepancreatic endoderm, cells at the endoderm stage were fed for $24 \mathrm{~h}$ with Advanced DMEM with B-27 supplement without vitamin A, 1\% Glutamax, $500 \mathrm{nM}$ retinoic acid (Calbiochem), $50 \mathrm{nM} \mathrm{A-83-01} \mathrm{(Calbiochem)} \mathrm{and} 8 \mathrm{ng} / \mathrm{ml}$ Bmp4 (Stemgent). For mesodermal differentiation, cells at the mesendoderm stage were treated for $48 \mathrm{~h}$ with $10 \mathrm{ng} / \mathrm{ml} \mathrm{Bmp} 4$.

ESCs with doxycycline-inducible alleles for Sox2, Foxa1, $\operatorname{Mnf} 1 \beta, \mathrm{Cdx} 2$, Gata6, Zfp161 and Klf7 in the HPRT locus were created as described ${ }^{48}$ and maintained and differentiated as above. For dominant negative lines, DNAbinding domains of NFYA and Nrf1 were used to create doxycycline-inducible HPRT lines as above.

Dominant negative lines were grown for $>7 \mathrm{~d}$ in $\mathrm{mES}$ medium supplemented with $5 \mathrm{nM}$ GSK-3 inhibitor XV and $500 \mathrm{nM}$ UO126 to enhance pluripotency ${ }^{49}$ and $2 \mu \mathrm{g} / \mathrm{ml}$ doxycycline. Cells were harvested at this stage for DNase-qPCR. For ChIP-qPCR, cells were treated for $6 \mathrm{~h}$ with mES medium with $1 \mu \mathrm{M}$ retinoic acid.

Tol2 GFP reporter transposon construct generation, transfection and flow cytometry. PCR-amplified constructs containing pioneer and nonpioneer motif regions and RXR:RAR binding sites were generated from primers listed below and cloned into PacI and AscI sites of p2TAL200R175-minHsp-GFP-BlR (R.I.S., S.L., C.W.O., J.P.v.H., P. Rolfe, K. Kawakami et al.; unpublished data). To generate the reporter construct with $2-\mathrm{kb}$ spacer DNA added between the enhancer and promoter, $2 \mathrm{~kb}$ of genomic DNA from a consistently DNase I-insensitive genomic region (primers are listed in Supplementary Table 5) was cloned into the PacI site of p2TAL200R175-minHsp-GFP-BlR.

Tol2-containing reporter plasmids and transposase-containing pCAGGSmT2TP (R.I.S., S.L., C.W.O., J.P.v.H., P. Rolfe, K. Kawakami et al.; unpublished data) were transfected into the mES lines using Xfect for mESCs transfection reagent (Clontech). Blasticidin selection was performed for $>7 \mathrm{~d}$ in mESC medium with 5 nM GSK-3 inhibitor XV and $500 \mathrm{nM}$ UO126 added to enhance pluripotency 49 .
For detection of GFP by flow cytometry, cells were trypsinized and seeded at $3 \times 10^{4}$ cells $/ \mathrm{cm}^{2}$ onto 96 -well plates. Cells were treated with mESC medium alone or supplemented with $1 \mu \mathrm{M}$ retinoic acid and/or $2 \mu \mathrm{g} / \mathrm{ml}$ doxycycline or differentiated into mesendoderm before treatment. After $24 \mathrm{~h}$, cells were trypsinized and quenched, and fluorescence of $5 \times 10^{3}$ to $20 \times 10^{3}$ cells was measured using a BD Accuri C6 flow cytometer and accompanying software (BD Biosciences).

Antibodies and immunofluorescence analysis. For cell immunofluorescence analysis, tissue-culture plates were fixed for $20 \mathrm{~min}$ in $4 \%$ paraformaldehyde (Electron Microscopy Sciences) and washed in PBS with $0.1 \%$ Triton X-100 (Sigma). Tissues were blocked by $20 \mathrm{~min}$ incubation at $4{ }^{\circ} \mathrm{C}$ in PBS with $20 \%$ donkey serum (Jackson ImmunoResearch) and $0.1 \%$ Triton X-100. Primary and secondary antibody staining were performed overnight at $4{ }^{\circ} \mathrm{C}$ in PBS with $5 \%$ donkey serum and $0.1 \%$ Triton $\mathrm{X}-100$, and after primary and secondary antibody staining, washing was performed with PBS with $0.1 \%$ Triton X-100. After staining, plates were washed and incubated with $1 \mu \mathrm{g} / \mathrm{ml}$ Hoechst 33342 (Life Technologies). Imaging was performed using a DMI 6000b inverted fluorescence microscope (Leica), and image analysis was performed with the Leica AF6000 software.

The following primary antibodies were used: goat anti-Foxa2 M-20, rabbit anti-RAR M-454, rabbit anti-cMyc N-262 (Santa Cruz Biotechnology), rabbit anti-Foxa2 (Millipore); goat anti-Sox17, mouse anti-Sox2, (R\&D Systems); mouse anti-Hnf1 $\beta$ (BD Biosciences). Alexa Fluor 488 and Alexa Fluor 594 conjugates (Jackson ImmunoResearch) were used for secondary detection.

ChIP-qPCR. ChIP was performed according to the 'mammalian ChIP-onchip' protocol (Agilent). $1 \times 10^{7}$ to $5 \times 10^{7}$ cells were used for each experiment. qPCR primers are listed in Supplementary Table 5.

Oligonucleotides. Oligonucleotides used in this work are presented in Supplementary Table 5.

DNase-seq. DNase-seq was performed using adaptations of previous protocols ${ }^{50}$. A detailed protocol is available in Supplementary Methods.

DNase-qPCR. DNase-qPCR samples were prepared from the doxycylineinduced dominant-negative cell lines and control cell lines in the absence of doxycyline as per the DNase-seq protocol above. Experimental primers were designed for pioneer transcription factor binding sites and used in conjunction with the positive and negative hypersensitivity control primers described above in qPCR analyses. Hypersensitivity at experimental primers sites was calculated for the dominant negative lines and control lines as follows:

$$
\begin{aligned}
& \left.2^{(\text {average } \mathrm{Ct}}(\text { negative control primers })-\mathrm{Ct}_{(\text {experimental primer })}\right) \\
& -(\text { average } \mathrm{Ct} \text { (negative control primers) }- \text { average } \mathrm{Ct} \text { (positive control primers) })
\end{aligned}
$$

Significance was calculated using Student's $t$-test.

48. lacovino, M. et al. A conserved role for Hox paralog group 4 in regulation of hematopoietic progenitors. Stem Cells Dev. 18, 783-792 (2009).

49. Ying, Q.L. et al. The ground state of embryonic stem cell self-renewal. Nature 453, 519-523 (2008).

50. Song, L. \& Crawford, G.E. DNase-seq: a high-resolution technique for mapping active gene regulatory elements across the genome from mammalian cells. Cold Spring Harb. Protoc. 2010, prot5384 (2010). 